\title{
Strategies for prevention, control and eradication of zoonotic diseases
}

\author{
- CHANDRA SHEKHAR
}

\begin{abstract}
Zoonotic diseases have a significant effect on public health worldwide, showing a higher incidence rate in developing countries due to lack of control strategies, as well as the lack of education in the communities. About 75 per cent of emerging infectious disease in humans originates from animals. There are many factors that favor the spread of zoonotic diseases include change in farming practices; social, cultural, religious and lifestyle habits; rapid population growth; increasing poverty and urban migration; more frequent movement across international boundaries by tourists, workers, immigrants, and refugees; alterations in the habitats of animals and arthropod vectors that transmit disease; increasing numbers of persons with impaired host defenses; and changes in the way that food is processed and distributed. Therefore, collaboration between medical and veterinary scientists as well as public health practitioners and laboratory scientists is essential in order to investigate new and emerging zoonotic diseases. Fully equipped laboratories that contain subtyping technique tools are also essential to detect disease outbreaks and characterizing transmission routes. By using molecular subtyping, different strains can be differentiated based on their genotypes and phenotypes. In addition to laboratory techniques, the work of epidemiologists, ecologists, and environmentalists is crucial in establishing patterns and preventing outbreaks of zoonotic diseases.
\end{abstract}

Key words : Zoonoses, Prevention, Control, Eradication, Elimination

How to cite this paper : Shekhar, Chandra (2017). Strategies for prevention, control and eradication of zoonotic diseases. Vet. Sci. Res. J., 8(1\&2) : 54-63, DOI : 10.15740/HAS/VSRJ/8.1and2/54-63.

Paper History : Received : 07.03.2017; Accepted : 25.09.2017 